\title{
Tuning of Discretization in Bimatrix Game Approach to Power System Market Analysis
}

\author{
Kwang-Ho Lee, Member, IEEE \\ Dankook University \\ Seoul 140-714, Korea
}

\author{
Ross Baldick, Member, IEEE \\ The University of Texas \\ Austin, TX 78712
}

\begin{abstract}
An important aspect of the study of power system markets involves the assessment of strategic behavior of participants for maximizing their profits. In models of imperfect competition of a deregulated system, the key task is to find the Nash equilibrium. In this paper, the bimatrix approach for finding Nash equilibria is investigated. This approach determines pure and mixed equilibria using the complementary pivot algorithm. The mixed equilibrium in the matrix approach has the equal number of non-zeros property. This property makes it difficult to reproduce a smooth continuous distribution for the mixed equilibrium. This paper proposes an algorithm for adjusting the quantization value of discretization to reconstruct a continuous distribution from a discrete one.
\end{abstract}

Index Terms-Bidding, Bimatrix Game, Complementary Pivot Algorithm, Complementarity Problem, Discretization, Market Analysis, Mixed Equilibrium, Payoff Matrix Approach.

\section{INTRODUCTION}

$\mathrm{E}$ lectric power industries throughout the world are being restructured. The major goal of this restructuring is lowering prices through harnessing competition. However, the electric power industry has the characteristics of an oligopoly with imperfect competition, so there is the possibility that generating companies exercise market power. In the England and Wales market in 1990, the two larger companies were able to wield market power by selling power at considerably higher prices than their marginal cost [1].

The study of market power issues involves the assessment of strategic behavior of participants for maximizing their profits. In order to analyze the transactions and bidding strategies, many papers [2]-[8] use game theory as a rigorous tool. The strategies of participants for maximizing their profits can be formulated as an optimization problem. However, in a deregulated system the formulation is different from that in a centralized system. The individual objectives of participants are dependent on others' strategies, so that the participant profit maximization problem is constrained by the equilibrium conditions [16].

There are several alternative models of imperfect competition, such as the Cournot, Stackelberg, Bertrand, and supply function models. However, in each model the key task is to find the Nash equilibrium [9]. Solution methods for finding Nash equilibria can be categorized into four methods: the mathematical programming approach, co-evolutionary programming, exhaustive search, and the payoff matrix approach.

In [3]-[5], a mathematical programming approach is used to seek a Nash equilibrium. However, in those formulations, the overall profit functions are not proved to be concave, so the results of applying a local search algorithm might not be a Nash equilibrium. Moreover, these approaches do not seem to be able to identify if a pure strategy equilibrium fails to exist, and do not appear to be able to directly solve for mixed strategy equilibria.

In [3], a probabilistic distribution for a mixed strategy is constructed by combining points from a limit cycle of calculations in a mathematical programming approach. But when the mixed strategy has a distribution that involves nonzero probabilities for many pure strategies, it is not clear how this method could detect and represent the limit cycle.

In [10], a co-evolutionary technique is used for finding the global solution. However, non-differentiability of the profit function when there are potentially binding constraints in an electricity market poses difficulties for this approach.

An exhaustive search of the best response functions is applied to a three-player game in [11]. However, exhaustive search requires considerable effort to search the best response function space, and is not well-suited to finding a mixed equilibrium.

A fundamentally different approach is to represent the profit function using payoff matrices in a bimatrix game [6][7]. The payoff matrix approach can find a global solution for the given matrices and can represent mixed strategy equilibria [12]. This approach, which is based on Lemke's algorithm [13], will be explored in this paper.

For solving multi-player games, the payoff matrix approach has a difficulty, since the conventional solution technique of Lemke's algorithm is confined to two player games. Although the mathematical programming approach can solve the multiplayer game problems in theory, there are few studies showing the solution of the multi-player game. This is apparently due to computational difficulties in solving for more than two players. Consequently, this theoretical superiority of the mathematical programming approach in treating multiple players may not be of significant practical value, particularly when mixed 
strategies are sought.

We propose a method for using the payoff matrix approach to analyze mixed strategy equilibria in a two player game. In order to apply the payoff matrix approach to a gaming problem, discretization of the continuous decision variable is required to create the payoff matrix for each player [7]. When the problem has a mixed equilibrium, the number of nonzero elements in the mixed equilibrium is equal for both players [14][15]. This "equal number of non-zeros" property may lead to a solution that does not closely represent the distribution of the mixed equilibrium in the absence of discretization. In this paper the equal number of non-zero elements property will be derived in a way that is simple and different from the original derivation [15]. Also, this paper proposes a tuning algorithm for obtaining a smooth distribution in mixed equilibrium using that property.

The outline of the paper is as follows. Section II describes the formulation of the bimatrix game and its solution using Lemke's algorithm. Section III describes the equal number of non-zeros property and examples of mixed equilibria. Section IV presents a proposed algorithm for reconstructing a continuous distribution and examples of tuning the discretization, and section $\mathrm{V}$ presents the conclusion.

\section{BIMATRIX GAME}

\section{A. Equilibrium Strategy}

A bimatrix game is a two-player, nonzero-sum matrix game. The two players are designated N1 and N2. Player N1 has $m$ pure strategies, and $\mathrm{N} 2$ has $n$. In a Cournot model, for example, the strategies correspond to discretization of the continuous quantity variables. In a play, if N1 picks the pure strategy $i$ and $\mathrm{N} 2$ picks $j$, then the payoff to N1 is $a_{i j}$ and the payoff to $\mathrm{N} 2$ is $b_{i j}$. Define the $m \times n$ payoff matrices $A$ and $B$ by:

$$
A=\left[a_{i j}\right], \quad B=\left[b_{i j}\right] .
$$

Suppose N1 picks strategy $i$ with a probability of $x_{i}$. That is, the players are choosing mixed strategies. The column vector $x=\left(x_{i}\right) \in \mathrm{R}^{\mathrm{m}}$ completely defines N1's mixed strategy. Similarly let the probability vector $y=\left(y_{j}\right) \in \mathrm{R}^{\mathrm{n}}$ represent N2's mixed strategy. If $\mathrm{N} 1$ adopts strategy $x$ and $\mathrm{N} 2$ adopts strategy $y$, the expected payoffs to $\mathrm{N} 1$ and $\mathrm{N} 2$ are $x^{\mathrm{t}} A y$ and $x^{\mathrm{t}} B y$ respectively.

The strategy pair $\left(x^{*}, y^{*}\right)$ is said to be an equilibrium pair if no player benefits by unilaterally changing his own strategy while the other player's strategy is fixed. That is, if:

$$
x^{* \mathrm{t}} A y^{*} \geq x^{\mathrm{t}} A y^{*} \text {, for all } x \in \mathrm{R}^{\mathrm{m}} \text {, such that } x^{\mathrm{t}} e_{m}=1, x \geq 0,
$$

and $x^{* \mathrm{t}} B y^{*} \geq x^{* \mathrm{t}} B y$, for all $y \in \mathrm{R}^{\mathrm{n}}$, such that $y^{\mathrm{t}} e_{n}=1, y \geq 0$,

where $e_{m}$ and $e_{n}$ denote the column vectors in $\mathrm{R}^{\mathrm{m}}$ and $\mathrm{R}^{\mathrm{n}}$, respectively, in which all the elements are equal to 1 .

\section{B. Conversion to a Complementarity Problem}

The conditions for an equilibrium strategy, (1) and (2), can be converted into the form of a linear complementarity problem (LCP).
Let $E=e_{m} e_{n}{ }^{\mathrm{t}} \in \mathrm{R}^{\mathrm{m} \times \mathrm{n}}$ be the matrix of all ones. Let $k \in \mathrm{R}$ be fixed and large enough to satisfy $k E-B>0$ and $k E-A>0$. Then the conditions (1) and (2) are equivalent to ([13]):

$$
\begin{aligned}
& \bar{B}^{\mathrm{t}} x^{*}-e_{n} \geq 0, \quad x^{*} \geq 0, \text { and } y^{* \mathrm{t}}\left(\bar{B}^{\mathrm{t}} x^{*}-e_{n}\right)=0, \\
& \bar{A} y^{*}-e_{m} \geq 0, \quad y^{*} \geq 0, \text { and } x^{* \mathrm{t}}\left(\bar{A} y^{*}-e_{m}\right)=0,
\end{aligned}
$$

where $\bar{A}=k E-A$, and $\bar{B}=k E-B$.

Introducing slack variables corresponding to the inequalities in (3) and (4), the conditions for an equilibrium strategy are equivalent to:

$$
\begin{aligned}
& \left(\begin{array}{cc}
\underline{0} & \bar{B}^{\mathrm{t}} \\
\bar{A} & 0
\end{array}\right)\left(\begin{array}{c}
y^{*} \\
x^{*}
\end{array}\right)-\left(\begin{array}{l}
w^{*} \\
u^{*}
\end{array}\right)=\left(\begin{array}{c}
e_{n} \\
e_{m}
\end{array}\right), \\
& \left(\begin{array}{l}
y^{*} \\
x^{*}
\end{array}\right) \geq 0, \quad\left(\begin{array}{l}
w^{*} \\
u^{*}
\end{array}\right) \geq 0, \quad\left(\begin{array}{l}
y^{*} \\
x^{*}
\end{array}\right)^{\mathrm{t}}\left(\begin{array}{l}
w^{*} \\
u^{*}
\end{array}\right)=0,
\end{aligned}
$$

where $w$ and $u$ are slack variables.

Hence the conditions (1) and (2) of the equilibrium strategy are expressed as an LCP that has no objective function to be optimized. The LCP can be solved by a complementary pivot algorithm due to Lemke [13].

\section{MiXed EQuILIBRIUM}

\section{A. Property of mixed strategy}

Even though the matrix approach is adopted for approximating the mixed equilibrium by discretizing the continuous bidding parameter, the distribution of the mixed equilibrium is typically continuous since the actual bidding parameter is continuous. In principle, if the discretization has captured the important features of the bidding parameter, then interpolation of the discrete distribution of the mixed solution can reconstruct a continuous distribution. However, a property of mixed equilibria sometimes makes the resulting interpolated continuous distribution unrealistic. In this paper, such cases are demonstrated and a tuning algorithm is proposed for approximating a continuous distribution.

Reference [15] shows that in a bimatrix game each solution has associated with it a square sub-matrix of the payoff matrix corresponding to the non-zero entries in $x^{*}$ and $y^{*}$. Therefore $x^{*}$ and $y^{*}$ have the same number of nonzero elements. We call this the "equal number of non-zeros property." In the following, the property is derived in a different way from [15].

Lemke's algorithm can be described by a tableau method which is similar to the simplex tableau method for linear programming. In comparison with the simplex method, the LCP tableau method has a complementarity condition between two groups of variables $(x, y$ and $w, u)$, but has no objective function. The tableau of (5) for the augmented variable, $z=[w$, $u, y, x]$, can be expressed in the following $(m+n) \times 2(m+n)$ matrix form:

$$
\left(\begin{array}{cc:cc}
I_{n} & 0 & 0 & -\bar{B}^{\mathrm{t}} \\
0 & I_{m} & -\bar{A} & 0
\end{array}\right) .
$$


If an augmented variable is an equilibrium of the game, then the variable can be partitioned into basic and nonbasic variables, $z_{B}$ and $z_{D}$, respectively. Let $M_{B}$ be a basis matrix of the tableau matrix, which consists of the columns corresponding to the basic variables. $M_{B}$ is square and nonsingular. Rearrange $M_{B}$ to form the matrix $M_{B}^{\prime}$ as follows:

$$
M_{B}^{\prime}=\left(\begin{array}{cc}
M_{1} & 0 \\
0 & M_{2}
\end{array}\right) \text {. }
$$

The columns in $M_{l}$ correspond to the basic variables among the variables $w$ and $x$, and the columns in $M_{2}$ correspond to those among $u$ and $y$. In other words, $M_{l}$ is extracted from the submatrix $\left[I_{n},-\bar{B}^{\mathrm{t}}\right]$, and $M_{2}$ from $\left[I_{m},-\bar{A}\right]$.

Suppose that N1 has $p$ strategies as non-zero elements in his mixed equilibrium, and $\mathrm{N} 2$ has $q$. Then the numbers of basic variables that comes from the variables $w, u, y$, and $x$, are $n-q$, $m-p, q$, and $p$ respectively, since $w$ and $y$ are complementary to each other, and so are $u$ and $x$. Hence $M_{l}$ has a dimension of $n \times(n-q+p)$, and $M_{2}$ has that of $m \times(m-p+q)$.

Since the basis $M_{B}$ is non-singular, the sub-matrices, $M_{l}$ and $M_{2}$, are also non-singular. Since $M_{B}^{\prime}$ is block diagonal, $M_{I}$ and $M_{2}$ are $n \times n$, and $m \times m$ square matrices respectively. This leads to the property that $p$ equals $q$. That is, each player has equal number of strategies in the mixed equilibrium.

\section{B. Two bus example}

In the typical mathematical programming approach to solve the Nash equilibrium, the problem is formulated deterministically. If there is no pure strategy Nash equilibrium, then such an algorithm may oscillate in a limit cycle, or, converge to a "false equilibrium." In [3], a model of an electricity market with two firms and a transmission line is presented. The specification of the system is reproduced in Appendix A.1. One of the firms has a jump in its best response curve when a transmission line constraint is considered. Applying a mathematical programming approach results in a limit cycle.

The characteristics of the limit cycle are used to deduce the mixed strategy equilibrium in [3]. Player 1 has one pure strategy of bidding parameter $\mathrm{k} 1$, and player 2 has two candidates for parameter $\mathrm{k} 2$. The mixed equilibrium is $\mathrm{k} 1=1.372$ with probability 1.0 , and $\mathrm{k} 2=1.246$ with probability 0.56 and $\mathrm{k} 2=1.525$ with probability 0.44 . This distribution was found by searching the combination of two candidates for the best payoff.

However, if several pure strategies are represented in the mixed equilibrium, then detecting limit cycles and determining
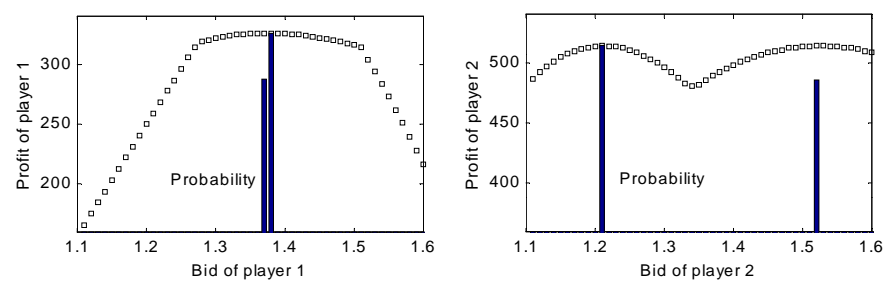

Fig. 1 Mixed equilibrium of the system in [3] the mixed strategy appears to be computationally prohibitive. We find a mixed equilibrium for the same problem by using the payoff matrix approach. The approach is also able to find mixed equilibria when there are many pure strategies represented in the mixed equilibrium.

Payoff matrices are constructed by discretization with $\Delta \mathrm{k}=0.01$, between $\mathrm{k}=1.11$ and $\mathrm{k}=1.60$. The mixed equilibrium is obtained using Lemke's algorithm, as follows:

$$
\begin{aligned}
& \mathrm{k} 1=1.37 \text { with probability } 0.4346 \\
& \mathrm{k} 1=1.38 \text { with probability } 0.5654 \\
& \mathrm{k} 2=1.21 \text { with probability } 0.5507 \\
& \mathrm{k} 2=1.52 \text { with probability } 0.4493
\end{aligned}
$$

By observing the profit for each player with the bids of the other player fixed, those strategies can be confirmed to be an equilibrium in the given payoff matrices. Figure 1 shows the profit curves for each player (shown as $\square$ ), given the values specified above for the other player's bids, together with the probabilities of the bids (shown as bars).

The mixed strategy has the equal number of non-zeros property. The matrix approach gives directly the probabilistic distribution in the mixed equilibrium of player 2 . However, the solution of the two strategies, $\mathrm{k} 1=1.37$ and 1.38 , in player 1 is different from that of mathematical programming approach [3] where player 1 plays a pure strategy. In section IV, it is shown that this result of $\mathrm{k} 1$ for figure $1 \mathrm{can}$ converge to a pure strategy between 1.37 and 1.38 by a tuning algorithm that refines the discretization.

\section{Three bus example}

In [7], a continuous distribution of a mixed equilibrium was reconstructed by interpolating the discrete distribution. The specification of the system is provided in Appendix A.2. A mixed equilibrium is obtained using discretization of the generation quantity in $1.0 \mathrm{MW}$ steps. In the equilibrium shown in Fig.2, each supplier has a distribution with a support of 31 consecutive pure strategies. So the distribution looks smooth, and it can be regarded as the discretization of a continuous distribution. The continuous distribution can be reconstructed by interpolation. Figure 3 shows the result of interpolating the
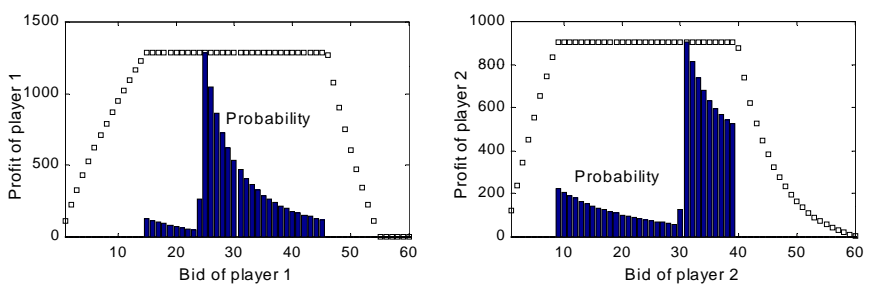

Fig. 2 Mixed equilibrium of the system in [7]
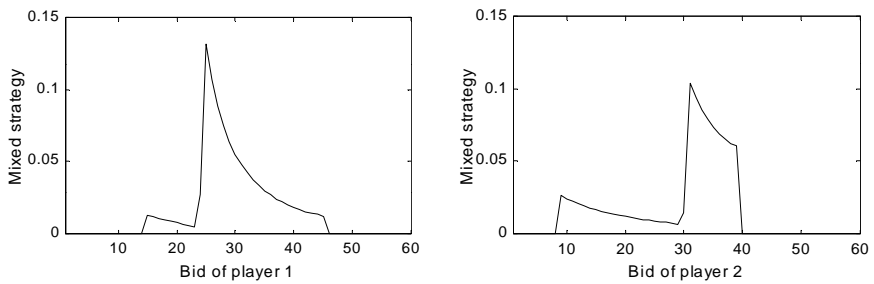

Fig. 3 Interpolated distribution from Fig.2 
results of Fig.2. This approximates the mixed strategy of the underlying continuous bid parameter.

However, the smoothness of a discrete distribution in the mixed equilibrium is not always guaranteed by the equal number of non-zeros property. The property may make it difficult to reconstruct a realistic continuous distribution. The successful reconstruction of a continuous distribution in this example is highly related to the discretization and the "support width" of both players. The support of a mixed strategy is the set of pure strategies which have positive values. The support width is computed by subtracting the ordinal number corresponding to minimum parameter from the ordinal number corresponding to maximum in the support. For example, in figure 2, the support width is $45-14=31$ for player 1 , and 39$8=31$ for player 2 .

\section{TUNING OF DISCRETIZATION}

\section{A. Difficulties in reconstructing a continuous distribution}

In the sample system [7], identical reactance is assumed in the three transmission lines. Suppose that we change the line parameters so that the reactance between bus 2 and bus 3 is twice the other two as given in Appendix. Then the congestion occurs at $\mathrm{q} 1-2 \cdot \mathrm{q} 2>20$ when Gen-1 is cheaper, and $2 \cdot \mathrm{q} 2-\mathrm{q} 1>20$ when Gen-2 is cheaper. Other assumptions are the same as those in [7]. Simulation is performed by again discretizing the generation quantity by $1.0 \mathrm{MW}$, from $1 \mathrm{MW}$ to $70 \mathrm{MW}$ in Gen-1 and $1 \mathrm{MW}$ to $50 \mathrm{MW}$ in Gen-2. So the payoff matrices have a dimension of $70 \times 50$. The results are shown in Fig. 4 with distributions of a mixed equilibrium and the expected profit for each supplier.

The number of strategies in this mixed equilibrium is 21 which is identical for each supplier. However, the distribution of player1's strategy is not smooth, but castellated, and the distribution of the expected profit has a ripple in the vicinity of its maximum. The support width of player 1 is $63-23=40$ while the support width of player 2 is $27-7=20$. If an interpolation is performed on Gen-1's distribution, it produces a sawtooth as shown in Fig.5. The sawtooth does not represent the mixed
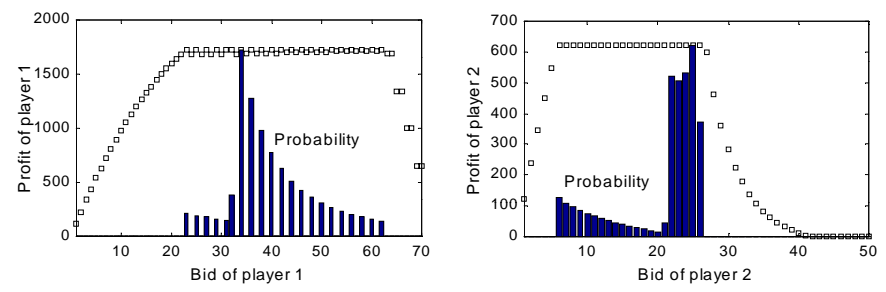

Fig. 4 Mixed Equilibrium of the modified system from [7]
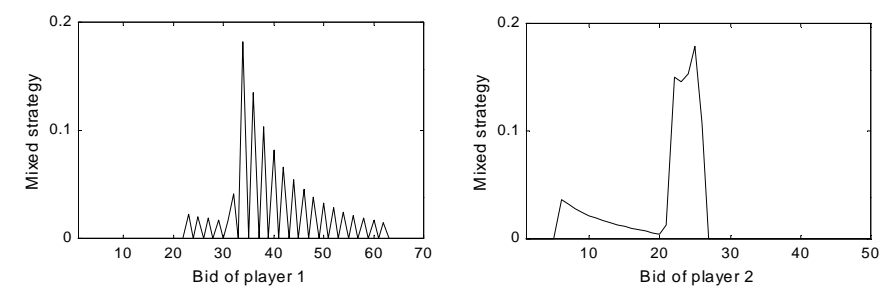

Fig. 5 Interpolated distribution from Fig.4 strategy of the underlying continuous bid parameter. It is an artifact of the discretization.

\section{B. Tuning algorithm for smooth and continuous distribution}

In order to get a smooth and continuous distribution of a mixed equilibrium, we adjust the discretization based on the support width of each player. If the support width in each player is not identical in a mixed equilibrium, then the discretization in each player's decision parameter needs to be adjusted to get a smooth and continuous distribution. In particular, the ratio of the discretizations should be changed by the ratio of the support widths for the two players. If the support width is known in advance, the mixed equilibrium of the smooth distribution is obtained directly with the adjusted discretization. However, when it is not known in advance, an adjustment of the discretization value is performed iteratively.

\section{Three bus example}

In the example system of section III.C, if a finer discretization of $0.5 \mathrm{MW}$ is used for both players, then the distribution of player 1 remains castellated as shown in Fig.6. In Fig.6, many blanks remain between the elements in player 1's support. Hence, a finer discretization does not necessarily produce a smoother distribution.

The support width of player 1's mixed equilibrium in Fig.4 is 40 (from 22 to 62), while that of player 2's is 20 (from 6 to 26). Since player 1's support width is twice that of player 2 , the discretization for player 2 needs to be twice as fine, or that for player 1 needs to be half (with the discretization of the other player fixed). Either case gives a smooth distribution. Fig.7 shows the result of the latter case with $1.0 \mathrm{MW}$ discretization for Gen-1, and $0.5 \mathrm{MW}$ for Gen-2.
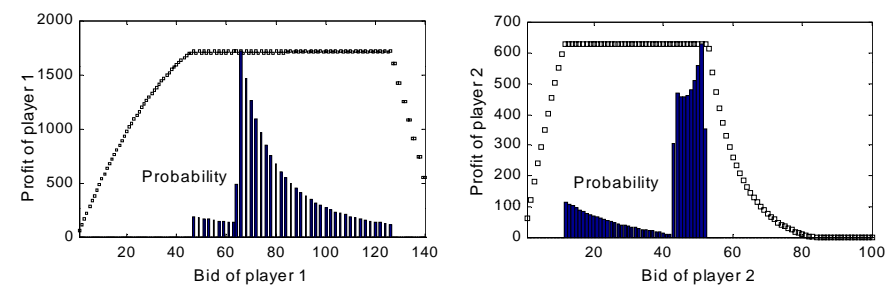

Fig. 6 Mixed equilibrium with finer discretization in both players
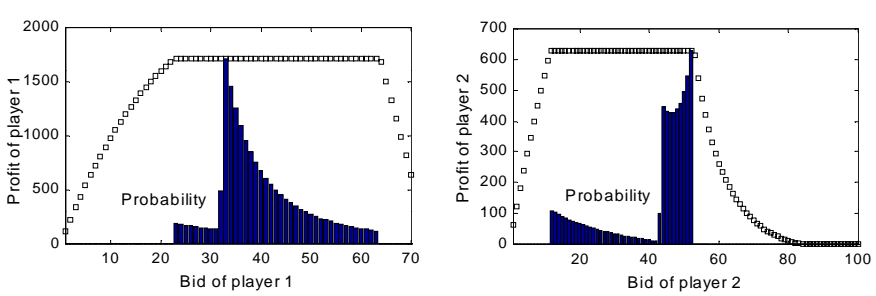

Fig. 7 Mixed equilibrium with adjusted discretization in player 2
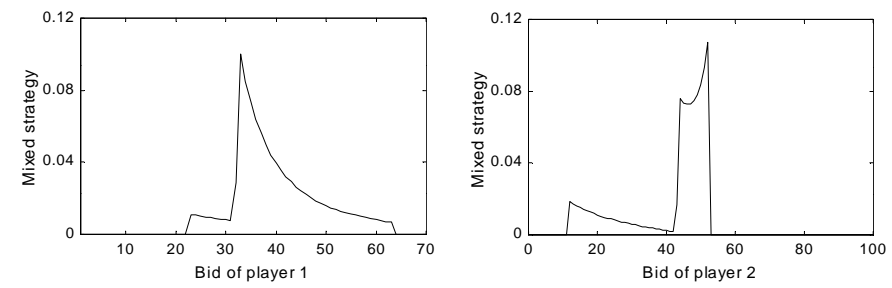

Fig. 8 Interpolated distribution from Fig.7 

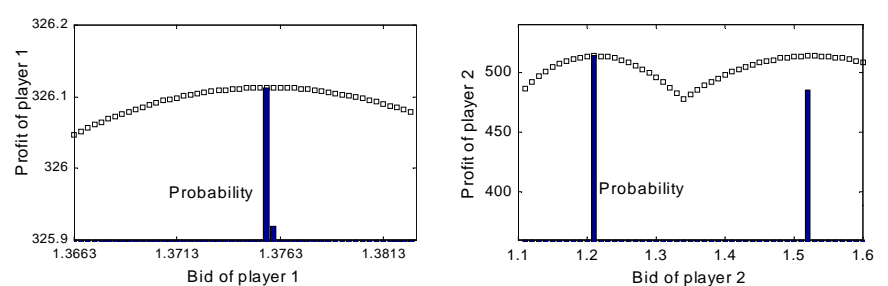

Fig. 9 Mixed equilibrium of finer version of Fig.1

As shown in Fig.7, each distribution is smooth without blanks between the elements in each support. After the smooth distributions in both players are obtained, the finer quantization that keeps the ratio equal to the ratio of support widths gives a smoother distribution that is closer to the continuous distribution. Fig. 8 shows the result of interpolating the results of Fig. 7. The sawtooth of Fig.5 has been eliminated and the interpolation approximates the mixed strategy for the underlying continuous bid parameter.

\section{Two bus example}

In the two bus example in section III.B, Fig. 1 shows different results from that represented in [3]. The matrix approach produces two strategies as player 1's mixed equilibrium, while the mathematical programming and limit cycle approach in [3] gives one pure strategy as player 1's mixed equilibrium. This difference can be resolved by applying the proposed algorithm to this problem. In figure 1, the support width for player 1 is 1 , while the support width for player 2 is 30 . The discretization of player 1 is adjusted to be 30 times finer, and the result is as shown in Fig.9. The opposite case of making the quantization coarser by 30 times for player 2 is discarded, since it is trivially unrealistic. The mixed equilibrium in Fig.9 is as follows:

$$
\begin{aligned}
& \mathrm{k} 1=1.3756 \text { with probability } 0.9151 \\
& \mathrm{k} 1=1.3759 \text { with probability } 0.0849 \\
& \mathrm{k} 2=1.21 \quad \text { with probability } 0.5507 \\
& \mathrm{k} 2=1.52 \quad \text { with probability } 0.4493
\end{aligned}
$$

After the algorithm is applied, the support widths using the finer discretization have the same ratio. So the adjustment of the discretization will be performed over again. As the process is repeated, the solution of player 1 converges to playing the pure strategy close to $1.3756^{1}$ with probability 1 . Hence, the matrix approach is shown to be able to converge to the mixed equilibrium where one player has a pure strategy and the other plays a mixture of two pure strategies.

\footnotetext{
1 This result differs very slightly from [3]. We believe that the difference is due to round-off errors.
}

\section{CONCLUSION}

The matrix approach, co-evolutionary programming, exhaustive search, and the mathematical programming approach have been used for solving Nash equilibria in power markets. The matrix approach provides pure and mixed equilibria by the complementary pivot algorithm, while the other approaches have difficulties in computing the mixed equilibrium.

The matrix approach requires discretization of the continuous decision parameter for constructing the payoff matrices. After a mixed equilibrium is obtained, its continuous distribution can be reconstructed by interpolating the discrete distribution. However, the mixed equilibrium has the equal number of non-zeros property that the number of elements in each support is equal. This property can produce blanks in the distribution of a mixed equilibrium that are not indicative of the underlying continuous distribution. In this case, interpolation of this castellated distribution does not reconstruct a smooth continuous distribution.

This paper proposes an algorithm for reconstructing a continuous distribution from the discrete one. By adjusting the quantization value of discretization to the support width in the mixed equilibrium, a smooth and continuous distribution can be reconstructed.

Even though the matrix approach is quite fast for finding Nash equilibria and is able to find mixed equilibria, it is restricted to two player games. The concept of a multi-player games is the same as that of bimatrix games, but an efficient solution algorithm for multi-player games has not been developed yet.

\section{REFERENCES}

[1] R.J. Green and D.M. Newbery, "Competition in the British Electricity Spot Market," The Journal of Political Economy, Volume 100, Issue 5, pp. 929-953, October 1992.

[2] X. Bai, S.M. Shahidehpour, V.C. Ramesh, and E. Yu, "Transmission Analysis by Nash Game Method," IEEE Trans. on Power Systems, Vol.12, No.3, pp. 1046-1052, August 1997.

[3] J.D. Weber and T.J. Overbye, "A Two-Level Optimization Problem for Analysis of Market Bidding Strategies," IEEE PES Summer Meeting, Vol.2, pp.682-687, 1999.

[4] B.F. Hobbs, C.B. Metzler, and J.S. Pang, "Strategic Gaming Analysis for Electric Power Systems: An MPEC approach," IEEE Trans. on Power Systems, Vol.15, No.2, pp. 638-645, May 2000.

[5] B.F. Hobbs, "Linear Complementarity Models of Nash-Cournot Competition in Bilateral and POOLCO Power Market," IEEE Trans. on Power Systems, Vol.16, No.2, pp.194-202, May 2001.

[6] R.W. Ferrero, S.M. Shahidehpour, and V.C. Ramesh, "Transaction Analysis in Deregulated Power Systems Using Game Theory," IEEE Trans. on Power Systems, Vol.12, No.3, pp.1340-1347, August 1997.

[7] S. Stoft, "Using Game Theory to Study Market Power in Simple Networks," IEEE Tutorial on Game Theory in Electric Power Markets, IEEE Press TP-136-0, pp.33-40, 1999.

[8] X. Guan, Y.C. Ho, D.L. Pepyne, "Gaming and Price Spikes in Electric Power Markets," IEEE Trans. on Power Systems, Vol.16, No.3, pp.402408, August 2001.

[9] D.W. Carlton, J.M. Perloff, Modern Industrial Organization, AddisonWesley, 2000.

[10] T. Curzon Price, "Using Co-evolutionary Programming to Simulate Strategic Behavior in Markets," Journal of Evolutionary Economics, Vol.7, pp.219-254, 1997. 
[11] L.B. Cunningham, R. Baldick, and M.L. Baughman, "An Empirical Study of Applied Game Theory: Transmission Constrained Cournot Behavior," to appear in IEEE Trans. Power Systems, 2001.

[12] D. Fudenberg and J. Tirole, Game Theory, The MIT Press, 1991.

[13] C.E. Lemke and J.T. Howson, "Equilibrium Points of Bimatrix Games," SIAM Journal of Applied Mathematics 12, pp.413-423, 1964.

[14] L.S. Shapley, R.N. Snow, "Basic Solutions of Discrete Games," Contributions to the Theory of Games, Vol.I, Princeton, pp. 27-35, 1950.

[15] N.N. Vorob'ev, "Equilibrium Points in Bimatrix Games," Theory of Probability and its Applications, Vol.III, pp.297-309, 1958.

[16] Z.Q. Luo, J.S. Pang, and D. Ralph, Mathematical Programs with Equilibrium Constraints, N.Y.: Cambridge Univ. Press, 1996.

\section{BIOGRAPHIES}

Kwang-Ho Lee was born in Seoul, Korea 1965. He received his B.S., M. S., and Ph.D. degrees from Seoul National University in 1988, 1990, and 1995, respectively, in electrical engineering. He conducted research on Reliability Enhancement of power systems in the Korea Electrical Power Research Institute. $\mathrm{He}$ is presently an Assistant Professor in the Department of Electrical Engineering at Dankook University, Seoul, Korea.

Ross Baldick received his B.Sc. and B.E. from the University of Sydney, Australia and his M.S. and Ph.D. from the University of California, Berkeley. From 1991-1992 he was a post-doctoral fellow at the Lawrence Berkeley Laboratory. In 1992 and 1993 he was an Assistant Professor at Worcestor Polytechnic Institute. $\mathrm{He}$ is currently an Associate Professor in the Department of Electrical and Computer Engineering at the University of Texas at Austin.

\section{APPENDIX (SAMPLE SYSTEM MODEL)}

\section{A.1 Two-Bus Model (Supply function equilibrium) [3]}

Supply function of G1: $\mathrm{C}\left(s_{1}\right)=k_{1}\left(0.01 s_{1}{ }^{2}+10 s_{1}\right)$

Supply function of G2: $\mathrm{C}\left(s_{2}\right)=k_{2}\left(0.01 s_{2}{ }^{2}+10 s_{2}\right)$

Bidding parameters: $k_{1}, k_{2}$

Demand function of D2: $\mathrm{B}\left(d_{2}\right)=-0.04 d_{2}{ }^{2}+30 d_{2}$

Constraint on the transmission line: $80 \mathrm{MW}$

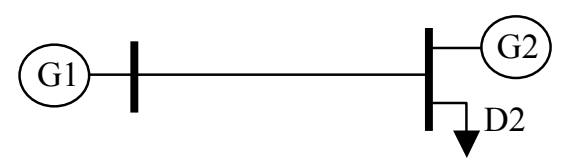

A.2 Three-Bus Model (Cournot Nash equilibrium) [7]

Constant marginal cost of G1: \$20/MWh

Constant marginal cost of G2: $\$ 40 / \mathrm{MWh}$

Demand function of D3: $\mathrm{B}\left(d_{3}\right)=-0.25 d_{3}{ }^{2}+100 d_{3}$

Constraint on the line $\mathrm{x}_{23}: 5 \mathrm{MW}$

Reactance on the lines:

(1) $\mathrm{x}_{12}=\mathrm{x}_{13}=\mathrm{x}_{23}$ (in the original reference and used in section III. $B$ of this paper)

(2) $\mathrm{x}_{12}=\mathrm{x}_{13}=1 / 2 \cdot \mathrm{x}_{23}$ (modified for discussion in section IV.A of this paper)

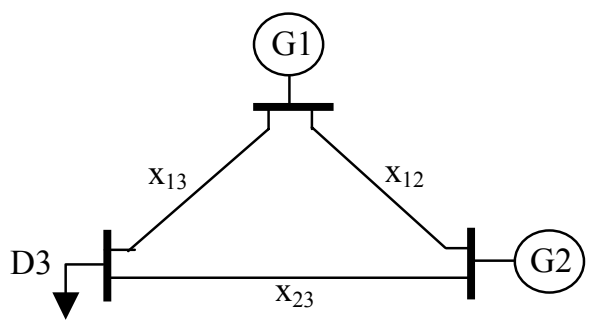

